\title{
Prospective increase in the training chance for nurses to learn radiation
}

\author{
Nami Yoshida, Yoshimasa Miyazaki and Mitsuru Nenoi* \\ Human Resources Development Center, National Institute of Radiological Sciences, National Institute for Quantum and Radiological Science and Technology, \\ Japan
}

\begin{abstract}
We expect that the nurses will be increasingly required to have enough knowledge on radiation because of the following reasons. First, an amazing technical innovation in radiation medicine causes elevation in the number of patients who are applied to radiation medicine either nuclear medicine or radiation cancer therapy. Second, the role of nurses in radiation emergency medicine is increasingly emphasized in response to the Fukushima-Daiichi Nuclear Power Plant accident. Finally, obedience to nursing ethics which places the highest priority on comfort of patients sometimes discourage the nurses against taking a correct action to protect themselves from unnecessary exposure to radiation. Based on these situational analyses, we expect that the demand for the training chance for nurses to learn radiation will increase, and the medical society will be requested to spend much money to establish more training facilities to encourage nurses to learn radiation.
\end{abstract}

The Human Resources Development Center (HRDC) of National Institute for Quantum and Radiological Science and Technology (QST) in Japan has offered a variety of training programs covering from basic knowledge to applied technologies in association with radiation [1]. The training course for radiobiology for nurses is a 5-day course which has been operated since 1994, aiming at providing basic knowledge of radiation for nurses or assistant nurses who are currently involved or planning to be involved in radiation medicine. The curriculum has been constantly reviewed and revised on a case-by-case basis. The latest curriculum is shown in Table 1 . We have experienced steady demand for the training course from hospitals and nursing schools all over the country [2]. As shown in Figure 1, the number of applicants fluctuated around 200 per year throughout the whole period of its history, while the number of trainees varied depending on the capacity of the HRDC. However, because of the current situation described below, we expect that the demand for the training chance for nurses to learn radiation will further increase in the coming decade.

First, the modern society witnesses an amazing technical innovation in radiation medicine, such as heavy ion radiotherapy with active beam shaping methods and diagnostic radiology by use of novel radiopharmaceuticals. Application of internal radiotherapy is also expected to expand based on tireless development of novel radioisotopelabeled drugs. The rapid progress in the technology in radiation medicine increases both the variety of radiation treatment protocols and the number of patients who are applicable to radiation medicine either nuclear medicine or radiation cancer therapy. In response to this trend, nurses will have to care more patients treated with a wide variety of novel protocols of radiation treatment. Therefore, a consistent effort of nurses to learn new nursing situation will be required.

Second, in response to the Fukushima-Daiichi Nuclear Power Plant accident, the role of nurses in radiation emergency medicine is increasingly underscored [3]. In the case of nuclear disasters, a large number of patients would need medical treatment and assistance of decontamination at hospitals. The nurses must be required not only for taking care of the patients' health but also for decontamination of patients, controlling radioactive contamination in the hospital as well as monitoring individual exposure to radiation of their own. Especially, mental care of the exposed patients who have anxiety about radiation hazards would be largely dependent on nurses' faceto-face communication. For these purposes, nurses should have basic knowledge on radiation and its risk.

Third, many nurses have a high professional moral which places the highest priority on comfort of patients. The so-called nursing ethics sometimes discourage the nurses against taking a correct action to protect themselves from unnecessary exposure to radiation. A

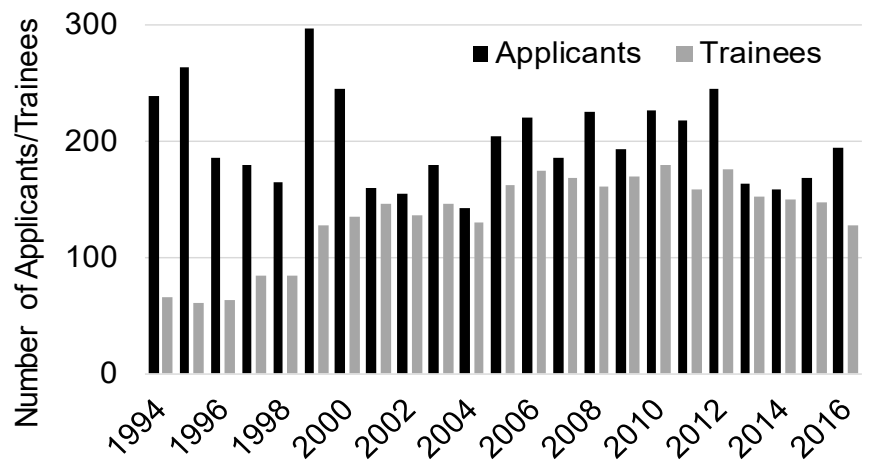

Figure 1: Time course change of the number of the applicants and trainees for the training course for radiobiology for nurses.

Correspondence to: Mitsuru Nenoi, Human Resources Development Center, National Institute of Radiological Sciences, National Institute for Quantum and Radiological Science and Technology, 4-9-1, Anagawa, Inage-ku Chiba 263-8555, Japan, Tel: +81-43-206-3049; Fax: +81-43-251-7819; E-mail: nenoi. mitsuru@qst.go.jp

key words: nursing, radiation, training

Received: June 03, 2017; Accepted: June 21, 2017; Published: June 23, 2017 
Table 1. Curriculum of the training course for radiobiology for nurses

\begin{tabular}{|l|l|}
\hline Lecture & \\
\hline Introduction to radiation & $70 \mathrm{~min}$ \\
\hline Radiation effects on human health & $80 \mathrm{~min}$ \\
\hline Basics of radiation therapy & $80 \mathrm{~min}$ \\
\hline Medical exposure and occupational exposure & $80 \mathrm{~min}$ \\
\hline Actual radiation protection in nursing scenes & $80 \mathrm{~min}$ \\
\hline Nursing care of radiation therapy patients & $150 \mathrm{~min}$ \\
\hline Radiopharmaceuticals & $80 \mathrm{~min}$ \\
\hline $\begin{array}{l}\text { Monitoring of radioactive contamination and } \\
\text { decontamination }\end{array}$ & $80 \mathrm{~min}$ \\
\hline Diagnostic imaging & $120 \mathrm{~min}$ \\
\hline Nursing care in diagnostic radiology & $80 \mathrm{~min}$ \\
\hline $\begin{array}{l}\text { Contribution of nurses at the Fukushima Daiichi } \\
\text { Nuclear Power Plant accident }\end{array}$ & $40 \mathrm{~min}$ \\
\hline Nursing care of interventional radiology patients & $120 \mathrm{~min}$ \\
\hline Mental care for diagnostic radiology patients & $120 \mathrm{~min}$ \\
\hline Practical Experiments/ Group Discussion & $60 \mathrm{~min}$ \\
\hline Radiation visualize by cloud chamber & $180 \mathrm{~min}$ \\
\hline $\begin{array}{l}\text { Tour of radiation facilities (Heavy Ion Medical } \\
\text { Accelerator, Radiation Emergency, Medicine } \\
\text { Facility, Diagnostic Imaging Facility, Hospital) }\end{array}$ & $140 \mathrm{~min}$ \\
\hline Characteristics of radiation and radiation protection & $140 \mathrm{~min}$ \\
\hline Individual exposure from X-ray photography & $120 \mathrm{~min}$ \\
\hline Group discussion on nursing in radiation medicine & \\
\hline
\end{tabular}

fundamental knowledge on protection of occupational exposure is definitely required for nurses to learn [4]. It should be emphasized that nurses need to understand that any quantity of radiation has carcinogenic potential depending on dose (known as the Linear NonThreshold Model). And the nurses should act on the optimization principle of radiation protection which rules that occupational exposure to radiation must be reduced as low as reasonably achievable (ALARA principle). The ALARA principle and the nursing ethics are not necessarily mutually exclusive. It must be possible for nurses to provide a nursing care of highest quality while avoiding unnecessary exposure to radiation at the same time.

Based on the circumstances described above, we suggest that the demand for the training chance for nurses to learn radiation will further increase. Therefore, we further suggest that the medical society should establish more training facilities to encourage nurses to learn radiation, and that international network should be constructed among training organizations to facilitate the mutual cooperation and improvement of curriculum quality.

\section{References}

1. http://www.nirs.qst.go.jp/information/training/invitation/index.html (in Japanese)

2. Shimizu Y, Iida H, Nenoi M (2017) Trends of training courses conducted in Human Resources Development Center of National Institute for Quantum and Radiological Science and Technology after the Fukushima Dai-ichi nuclear power plant accident. Health Phys 113: 66-70. [Crossref]

3. Hachiya M, Akashi M (2016) Lessons Learned from the Accident at the Fukushima Dai-ichi Nuclear Power Plant-More than Basic Knowledge: Education and its Effects Improve the Preparedness and Response to Radiation Emergency. Radiat Protect Dosim 171: 27-31.

4. Ohno K, Kaori T (2011) Effective education in radiation safety for nurses. Radiat Prot Dosimetry 147: 343-345. [Crossref]

Copyright: $\odot 2017$ Yoshida N. This is an open-access article distributed under the terms of the Creative Commons Attribution License, which permits unrestricted use, distribution, and reproduction in any medium, provided the original author and source are credited. 